\title{
Prediction of full field dynamic strain from limited sets of measured data
}

\author{
Peter Avitabile* and Pawan Pingle \\ Structural Dynamics and Acoustic Systems Laboratory, University of Massachusetts Lowell, MA, USA
}

\begin{abstract}
Dynamic response is an important consideration for design of structures due to operating or occasional loadings. The resulting dynamic stress strain is also of concern for fatigue and structural health monitoring. Typically, the actual loading and structural condition (boundary conditions, environmental condition, geometry, mechanical properties, etc.) are not necessarily known. Much effort is expended in attempting to identify the loads and appropriate model for prediction of these types of events. At best, the forces and actual boundary conditions are approximate and have an effect on the overall predicted response and resulting stress-strain that is identified for subsequent evaluation.

Experimental data can only be obtained from limited sets of points, such as those typically collected with accelerometers. These are normally used in the evaluation the state of a structure in service condition. More recently, Digital Image Correlation (DIC) and Dynamic Photogrammetry (DP) have become very important techniques to measure the surface response. These are non-contact and full-field techniques, which allow that much more simultaneous data to be measure.

The sets of limited surface data that are collected can be used in conjunction with an expansion algorithm to obtain full field information. The finite element model mass and stiffness matrices are used to obtain the normal constitutive relations as well as the modal characteristics. This information is used to develop the expansion algorithm and for the stress recovery during the back substitution process typically employed.
\end{abstract}

Keywords: Full field dynamic strain prediction, response prediction, dynamic response, full field response

\section{Introduction}

Structural systems are exposed to a variety of dynamic loading scenarios for which fatigue accumulation needs to be monitored and tracked over the course of the life of the structure. Generally, the loadings are of a level and occurrence to be considered normal routine loads and the design of the structure is built to accommodate these conditions. However, there are often other loadings which are more severe and are more important to the overall load and fatigue accumulation for the structure. These are also considered in the design of the structure but these loadings may be more significant overall. And many times the specific loading scenarios are determined using "best practices" or "best guesstimate" of the loading and its effect on the structure from a design standpoint. But the specific loading scenario is not accurately known and the actual load/response distribution is therefore not estimated correctly. Some common occurrences that could be considered more significant might be situations such as helicopter hard landing or a strong unexpected wind gust for a turbine blade or a barge/bridge strike to name a few rare situations that need to be more accurately depicted and understood in terms of the overall fatigue life of a structure.

In each of these cases, there are always "approximations" as to the severity of the loading or response, but the actual event characteristics are not well known or understood and therefore the estimated response and dynamic stress-strain conditions are not well known. In the case of the helicopter hard landing, the concern lies in the fact that an unknown portion of the fatigue life has been used up due to the event and it is unknown as to the overall effects on the available life remaining in the helicopter. For the wind turbine blade, strong winds may cause the blades to

\footnotetext{
*Corresponding author: Peter Avitabile, Structural Dynamics and Acoustic Systems Laboratory, University of Massachusetts Lowell, One University Avenue, Lowell, MA, USA. E-mail: peter_avitabile@uml.edu.
} 
deflect differently than the simulated loading developed with the finite element model and therefore the overall loading and resulting stress/strain distributions may be different than those approximated by the analytical model. And in the case of a barge strike, the prediction of the impact severity, distribution of load and the resulting response may be different than that which was simulated during the design process. These are just a very few examples of these occasional but very important loading scenarios. For each case described above, the underlying theme is the same. There are loads applied on the structural system that are not clearly known and/or understood. These misunderstood loads cause a displacement distribution which is different than those used for the design calculations. This, in turn, gives an inaccurate depiction of the fatigue life accumulated and the remaining life in the structure.

Minor or incidental loadings in the structure may not be critical to the overall life prediction. These low level events are likely to be reasonably well represented with the design analysis performed. However, the rare and more significant loadings may be of greater interest and need to better define them is important. The significant, rare, transient events might be very occasional but may cause damage or accumulate a significant portion of the fatigue life of the system. These types of events may have very limited sets of monitoring transducers which are insufficient to accurately depict the true stress-strain imposed on the structure. In order to assess the actual displacement and stress-strain distribution of the structure, more sophisticated utilization of the limited data available needs to be performed.

The most common approach used by many researchers is to approximate forces exciting the system through limited sets of measurements points. The application of those estimated forces to identify the finite element displacement and subsequently stress-strain distributions would be performed. This is a possible scenario but can be complicated with many approximations that render the solution to be very approximate. First, the estimated force at limited sets of degrees of freedom is very sensitive to the number of measurement points and the distribution of points on the structure [1]. Second, the finite element model needs to be accurately modeled and can accurately predict the actual full field displacement solution with the very coarse set of data points used to approximate the force applied on the structure; the resulting analysis and displacement distribution obtained can be very sensitive to these approximations. This approach is one possible alternative but is likely to have many difficulties in producing highly accurate results.

A different concept is proposed for the work presented herein. The ultimate goal is to provide a full field displacement that replicates the actual response obtained from the unknown loads applied/distributed on the structure. Rather than estimate the force, the approach here is to obtain accurate full field displacements from the limited sets of measurements, obtained from the actual system in operation. The expansion of real time operating response from limited sets of acquired data was recently studied by Chipman [2-4]. In that work, several expansion functions were developed to act as high order polynomial curvefitters based on an over-specification of an orthogonal set of modes that were expected to participate in the transient event. Chipman's work clearly showed that the data estimated from the expansion process was very accurate when compared to actual full field displacement measurements acquired for both analytical and experimental systems studied in that work. The current effort described here concentrates on using the finite element model and the over-specification of the set of modes that participate in the overall response as the set of orthogonal basis functions used to expand the limited set of measured data available for these very rare, short duration, but significant events.

An outline of the proposed approach utilized along with the pertinent theory is described in the next section with a schematic of the overall process used to generate the models used for full field dynamic stress-strain approximations. This is followed by a description of several test cases presented in this paper.

\section{Theory}

A common modeling approach relies on the general development of a finite element model, the assembly of the system matrices, the application of loads and boundary conditions followed by the solution of the set of equations and the recovery of the stress-strain solution. For many situations, the actual loading is not known and the actual boundary conditions are not well understood. This procedure is shown schematically in Fig. 1 for a wind turbine blade where significant loadings may occur while the system is in normal operation (i.e., rotating). 


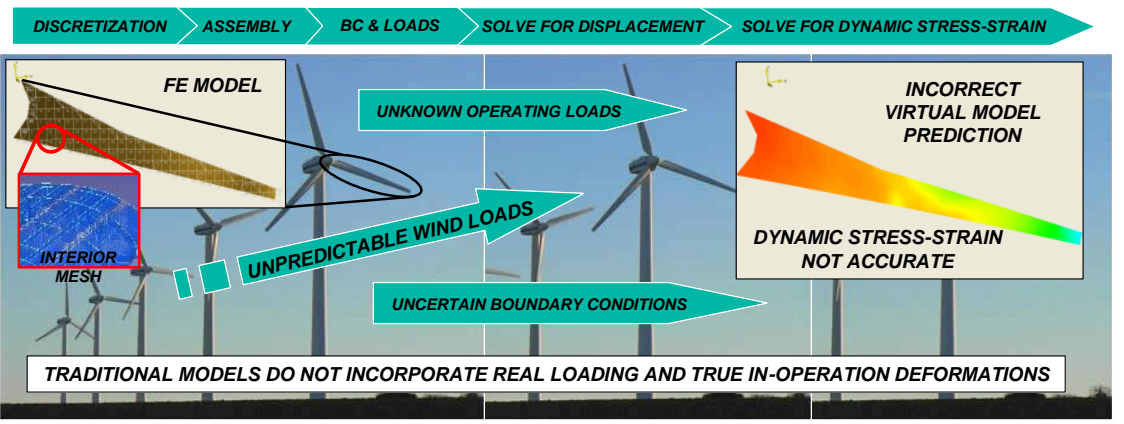

Fig. 1. Schematic showing normal finite element model development.

For the approach considered in this work, the difference is that the actual application of the loads and boundary conditions and the solution of the system set of equations are not specifically performed. Rather, the sparse set of displacements, measured from an actual operating event, is used with a set of orthogonal expansion functions to obtain the full field displacement solution [2-4]. This displacement solution is then used with the normal recovery of the stress-strain solution in the finite element modeling process [10,20-22]. This procedure is shown schematically in Fig. 2 where the limited set of measurement degrees of freedom are used with expansion processes to obtain the full field displacement for the system.

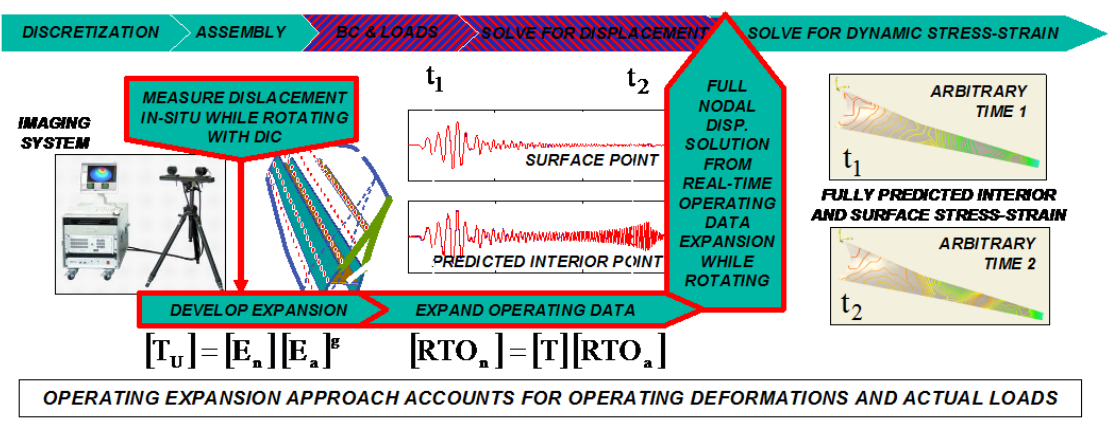

Fig. 2. Schematic showing alternation expansion/solution sequence.

With this approach, the finite element modeling solution process is intercepted and replaced with the expansion of the limited set of measured degrees of freedom. The actual expansion process has been presented in [2-4]. The basic theoretical approach is summarized below and utilizes concepts from model reduction and model expansion as the underlying methodology for the expansion approach used for this work.

\subsection{Model reduction and expansion}

Model reduction is necessary in order to develop expansion approaches for modal data for the unmeasured translational DOF (degree of freedom) as well as for rotational DOF. For this work the expansion is needed for augmenting the limited set of real-time operating data to provide a full field displacement solution. The reduction techniques are the basis of the expansion discussed in this work. These techniques have been presented in earlier work cited in the references; only summarizing equations are presented below. Several model reduction methods have commonly been used for expansion of measured data. Four common methods are Guyan [5], Dynamic Condensation [6], SEREP (System Equivalent Reduction-Expansion Process) [7], and a Hybrid method [8]. In these methods, the relationship between the full set of degrees of freedom and a reduced set of degrees of freedom can be written as 


$$
\left\{X_{n}\right\}=[T]\left\{X_{a}\right\}
$$

All of these methods require the formation of a transformation matrix that can project the full mass and stiffness matrices to a smaller size. The reduced matrices can be formulated as

$$
\begin{aligned}
& {\left[M_{a}\right]=[T]^{T}\left[M_{n}\right][T]} \\
& {\left[K_{a}\right]=[T]^{T}\left[K_{n}\right][T]}
\end{aligned}
$$

For the specific work in this paper, only the SEREP method has been used for the expansion of mode shapes. The System Equivalent Reduction Expansion Process (SEREP) produces reduced matrices for mass and stiffness that yield the exact frequencies and mode shapes as those obtained from the eigensolution of the full size matrix. The SEREP transformation is formed as

$$
\left[T_{U}\right]=\left[U_{n}\right]\left[U_{a}\right]^{g}
$$

The SEREP transformation is developed with analytical mode shapes for the structure (but can also be evaluated using measured modal vectors as done in Chipman's work [2-4]). Equation (1) is used for expansion of real-time operating data and is written as

$$
\left[\operatorname{ERTO}_{n}\right]=[T]\left[R T O_{a}\right]
$$

The transformation matrix, $[T]$, can be one of the matrices used for reduction described above but SEREP is used in all work presented herein.

The equations presented above summarize the critical equations necessary and have been documented in many different papers; these equations are developed in more detail in Appendix A. Tools for correlation of results using MAC and TRAC are described in Appendix B.

\section{Analytical simulations for displacement and strain}

In order to validate the methodology for the expansion technique to be used for determining displacement for dynamic stress and strain from limited sensor locations, an analytical study was performed on a model that resembles a blade of a wind turbine. The box-beam model used for analysis is shown in Fig. 3a and is assumed built in at the left face. The dimensions of the model are:

1. Length $(1)=60$ inches

2. Breadth $(b)=12$ inches

3. Width $(\mathrm{w})=6$ inches

4. Thickness of top and bottom plates (t1) $=0.5$ inches

5. Thickness of internal ribs $(\mathrm{t} 2)=0.25$ inches

6. Material - Aluminum

The finite element model of the box-shaped structure contains a total of 434 nodes. The natural frequencies and mode shapes of the beam are shown in Fig. 3b. The frequencies typically are well separated. The model has an assortment of modes containing transverse and lateral bending and torsion modes up to the first four modes which will be excited using a time pulse.

The excitation used was a time pulse applied vertically downwards, 10 inches from the bottom right corner of the box-beam. The input time pulse is shown in Fig. 4a. The time pulse is a combination of two triangular functions which when seen in frequency domain show a very uniform excitation over the frequency range of interest as shown in Fig. 4b. The time pulse primarily excites the first four modes of the structure as seen in FFT of the output response 
as shown in Fig. 4c. Modal damping typically seen in these types of structures was added to the model; a nominal value of $1 \%$ was used.

Many different cases have been studied in regards to the number of modes used for the expansion process, noise on the measurements and number of sensors used as described in $[10,20,21]$. There is much more data than that which can be presented in the scope of this paper. The full-field displacements obtained in [10,20-22] are used to generate full-field dynamic stress-strain solution in this section using procedure outlined previously in this paper. While noise and number of sensors have been studied, only the cases related to number of modes for expansion are shown here. Theoretically, there is no upper limit for the number of modes to be used for expansion, but from a practical standpoint only a limited number of modes are typically available for expansion.

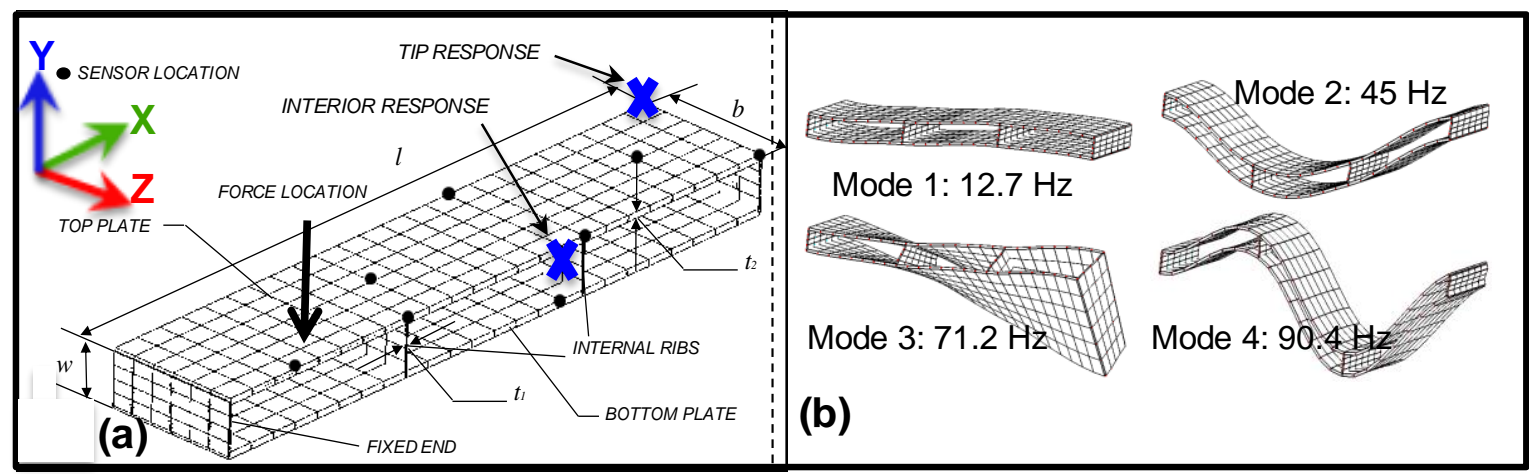

Fig. 3. (a) Box-beam model with the identification the position of force and the sparse sensors; (b) First four mode shapes of the structure.
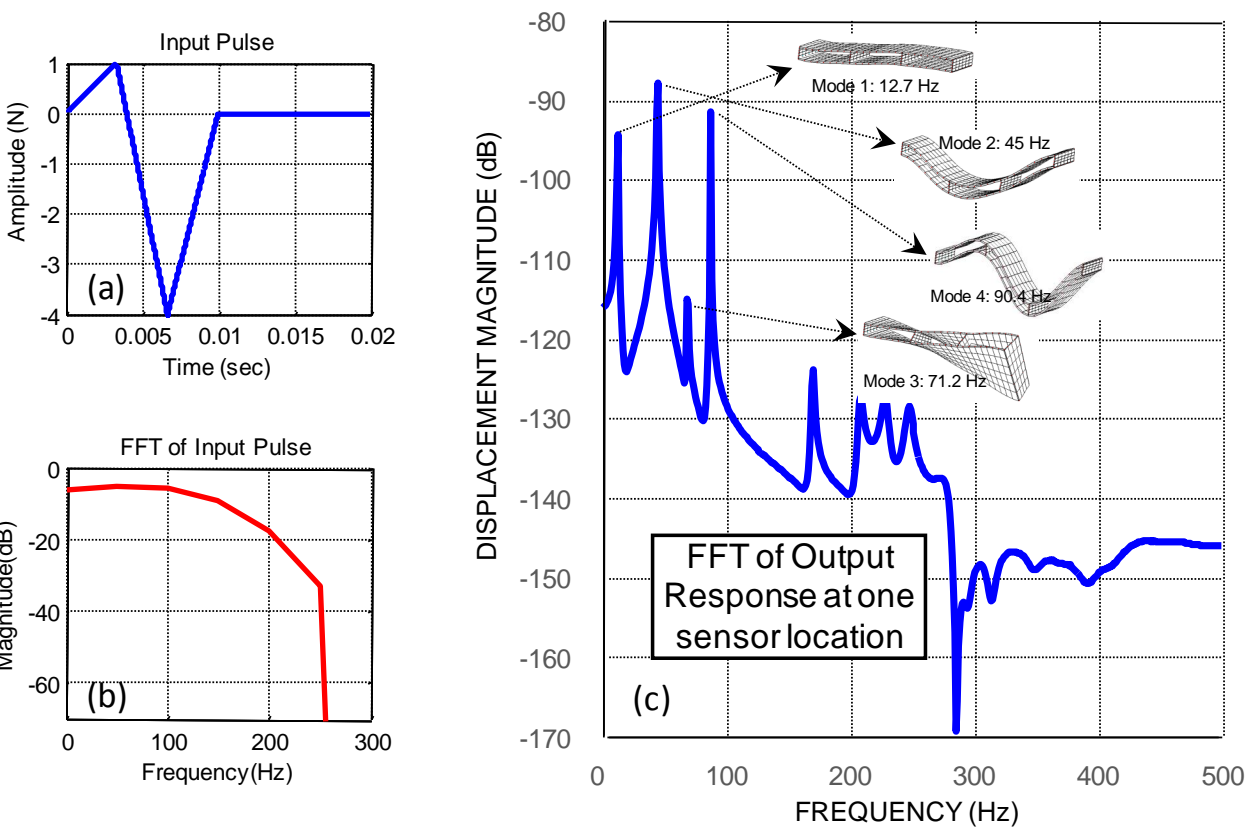

Fig. 4. Details of the excitation force and response of the structure.

In order to obtain useful results, the number of modes used in the expansion process are critically important. A sufficient number of modes that span the space of the solution must be included; this is true of any structural dy- 
namic modeling approach and is not a restriction for the technique. The transformation matrix used SEREP expansion technique where the transformation matrix is formulated using mode shape information. The expansion transformation matrix should contain at least those structure modes which are primarily excited. The different mode configurations studied in $[10,20,21]$ typically under-specified or over-specified the number of modes primarily excited by the forcing pulse. Because over-specifying the number of modes excited does not distort the solution, the full-field displacements obtained from over-specifying the number of modes excited will be used to obtain dynamic strain solution in this section.

When the first six modes were used in transformation matrix to obtain expanded full-field solution, the proposed approach compared well with the reference analytical displacement response solution; typical MAC and TRAC values were more than $98 \%$. The full-field displacements obtained from using first six modes are used for obtaining dynamic strain solution. This predicted dynamic strain solution (Y normal strain) is shown in comparison with reference strain solution in Figs $5 \mathrm{a}$ and $5 \mathrm{~b}$ with response location at the tip of the box-beam and in the rib of the box-beam respectively. Recall that the force pulse primarily excites the first four modes of the system. Only 14 y (vertical) direction points of a total of 434 finite element model nodes were used as sensor locations measuring vibration displacement response. The strain response is shown for a sample time period of 0.05 seconds. Although the full-field displacements obtained using first six modes provided excellent correlation with reference displacement solution, the full-field strain results obtained after incorporating the six-mode full-field displacement solution is not as good as expected (Figs 5a and b). However, when the full-field displacement solution obtained from using first ten modes is used to obtain the full-field dynamic stress-strain solution then excellent correlation is seen with reference strain solution as seen in Figs $6 \mathrm{a}$ and $6 \mathrm{~b}$. The strain solution therefore is more sensitive to mode configuration, in particular the contribution from higher modes, than the displacement solution. This trend is more prominent for higher strain regions (strain in the rib) as seen from Figs $5 \mathrm{~b}$ and $6 \mathrm{~b}$.

To study the sensitivity of strain solution in detail, the displacement solution correlation was studied. Figure 7 shows the MAC for displacement solution between the predicted and reference model responses for time period corresponding to the strain time period (0.2-0.25 seconds). The MAC plots are shown for three different mode configurations - using first six, eight and ten modes in expansion solution. The MAC for all three mode configurations is very good because they all over-specify the required number of modes for expansion. So to show the difference between six, eight and ten mode configuration, the MAC plots are shown from 0.9-1 indicating that the MAC for that particular time period is better than $90 \%$ always. Notice that even though with six-mode configuration, the displacement responses show good correlation (better than 90\%), the correlation however improves with eight and ten modes for expansion. In general, the results are very good and shows that the expansion process can produce acceptable prediction of the full field dynamic stress-strain for the structure from limited sets of measured data.

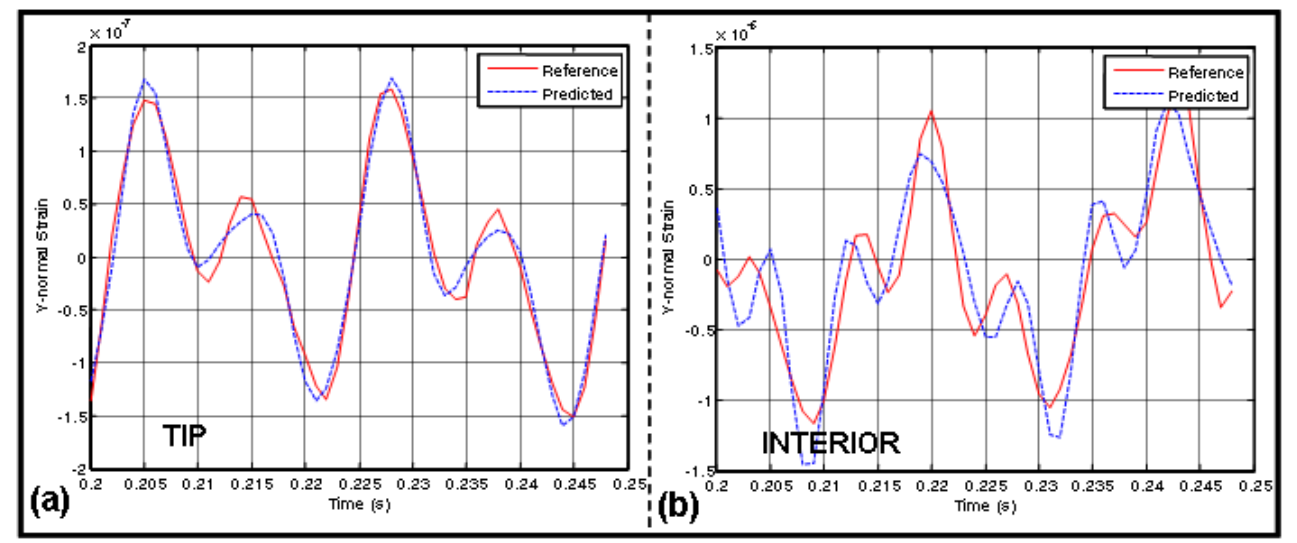

Fig. 5. Overlay of strain response of box-beam at (a) tip and (b) interior location; predicted solution used first 6 modes in expansion process. 


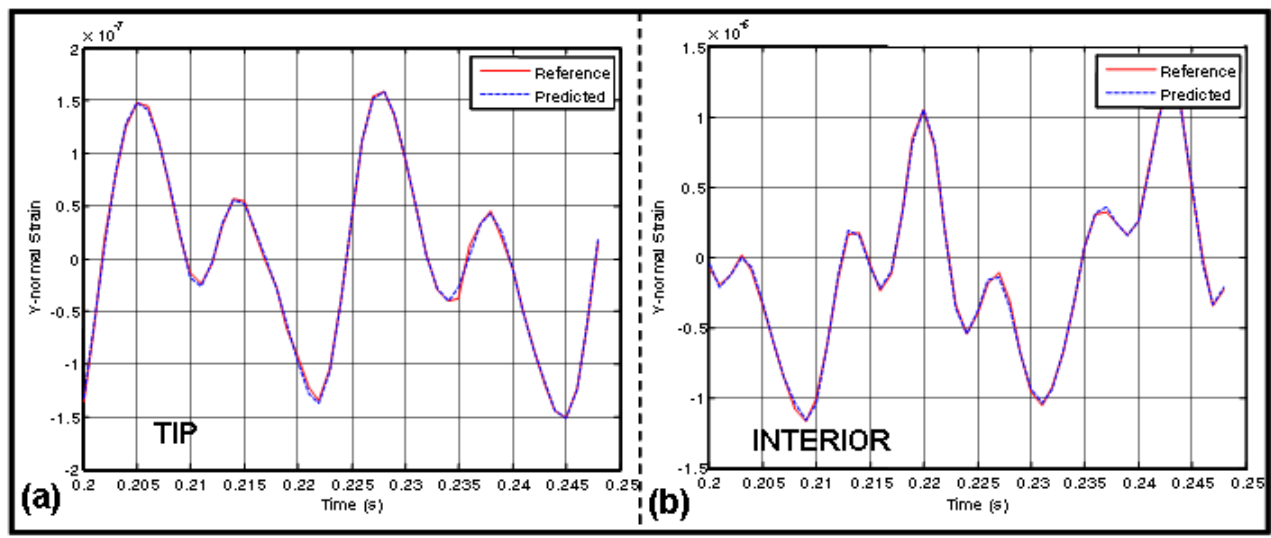

Fig. 6. Overlay of strain response of box-beam at (a) tip and (b) interior location; predicted solution used first 10 modes in expansion process.

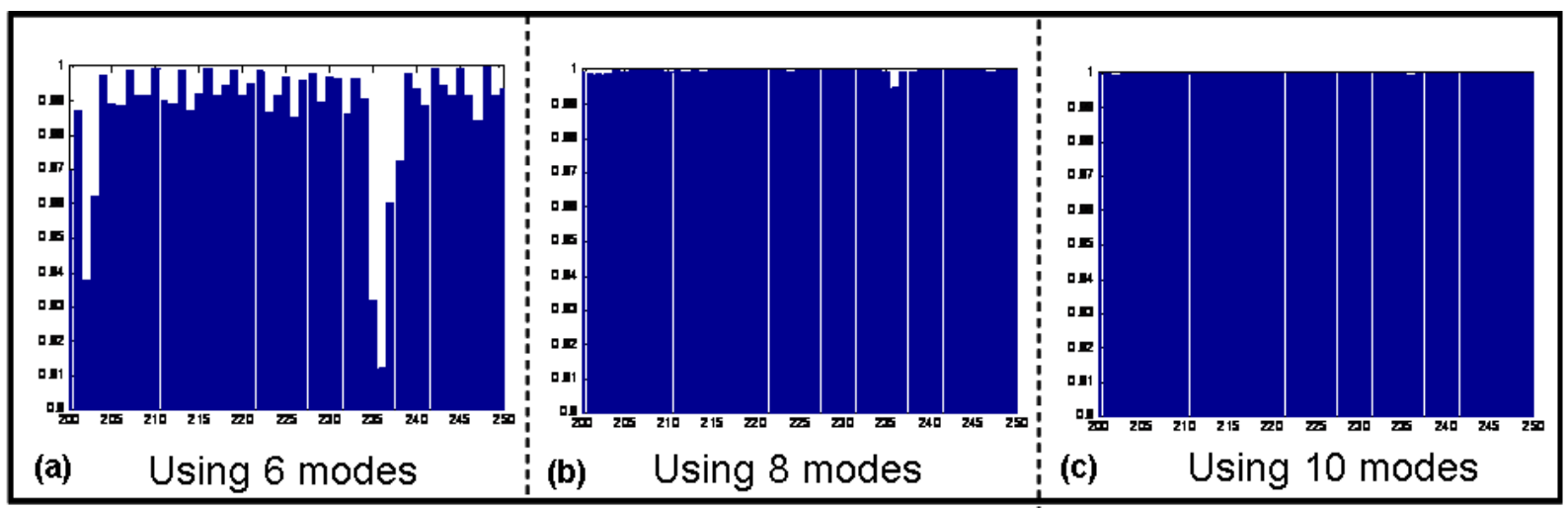

Fig. 7. MAC between displacement response vectors of reference and expansion algorithm solutions with different number of modes used for expansion, (a) using first 6 modes (b) using first 8 modes, (c) using first 10 modes in expansion process; MAC performed for time steps 200-250 and shown for $0.9-1$

\section{Experimental data for displacement and strain}

For this study, a simple structure (Base-Upright or BU), which has well correlated analytical and experimental modes from previous studies, was used. The structure was assembled from two aluminum plates. The structure has well-spaced, directional modes that could be identified easily. Figure 8 shows the BU with the primary dimensions labeled. The base plate is $24 \times 24$ inches and rigidly bolted to the tied to the ground at the four corners using concrete anchors, while the upright is $24 \times 30$ inches. Both plates are 3/4" thick aluminum. The base plate and the upright are held together by 0.25 inch thick, 1.5 inch wide, 24 inch long steel L-brackets using 0.5 inch bolts.

A Finite Element Model (FEM) of the BU is available and has been shown to be very well correlated to other measured test data from previous studies; detailed results of the correlation of the model to test data is presented in Appendix C. The model was developed using FEMAP [12] and other processing and manipulation of data was performed using MATLAB [12]. The BU FEM was created using plate elements for all the components and has approximately 58,000 DOFs. Rigid links were used to tie the L-brackets to the plates along the two main, 24 inch edges of the L-brackets. Solid cylindrical elements were used at the bolt locations with the appropriate mass to mimic the effect of the bolts. The BU FEM was fixed at the four locations corresponding to the points tied to the ground in the actual structure. An eigensolution was performed on the BU FEM to determine the mode shape information. For reference, the finite element model frequencies and mode shapes for the first 8 modes are shown in Fig. 9 for reference. 


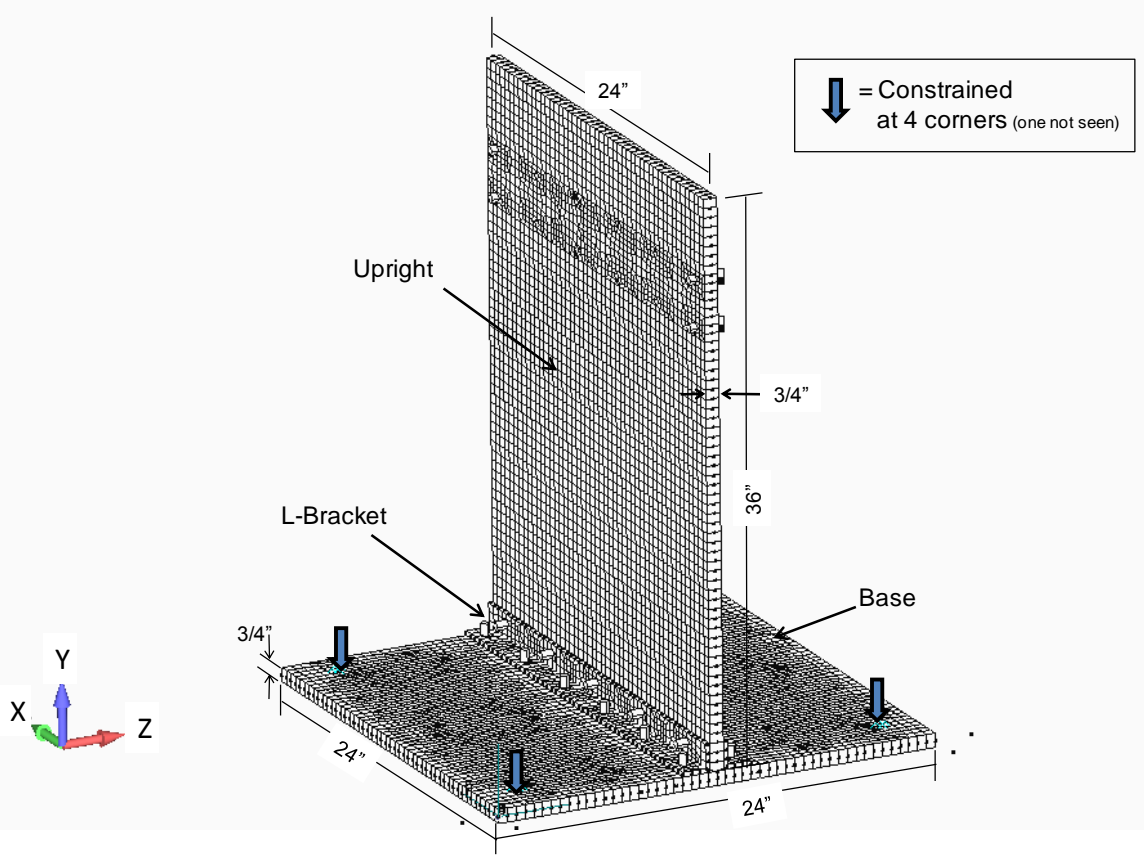

Fig. 8. Base-upright structure used for testing.

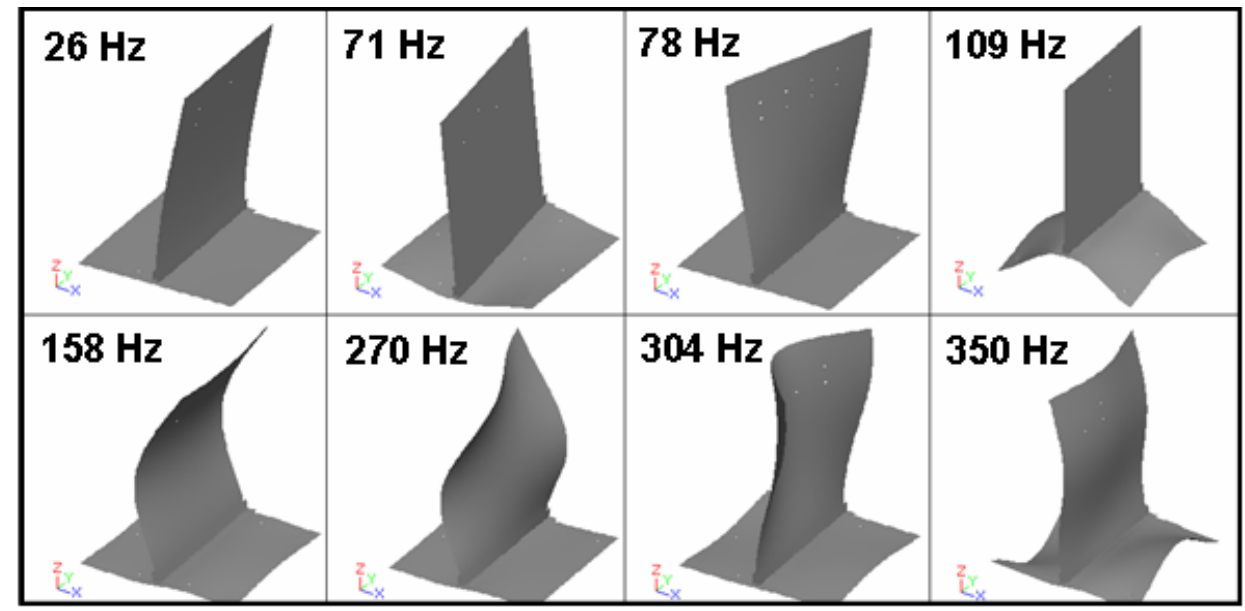

Fig. 9. First 8 mode shapes of the BU structure.

The displacement data is recorded at 8 patches using digital image correlation (ARAMIS [13]) technique and at 8 target locations using dynamic photogrammetry (PONTOS [14]) technique as shown in Fig. 10. The 8 targets used for PONTOS are located on top of the 8 ARAMIS patches. Both sets of data provide similar information and are provided for redundancy as well as comparison for the two similar but different techniques.

An impact with a calibrated modal hammer was made at the top-right corner at Location 1 in Fig. 10 of the Upright structure. The displacement data was collected at the same 8 target locations shown in Fig. 10. All data collected was acquired in the direction normal to the surface of the Upright. The displacement responses at 7 of the 8 recorded locations were used to expand to over 3000 finite element nodes using the expansion process. Displacement response was collected for 4 seconds using 800 frames per second cameras which means data was sampled at 


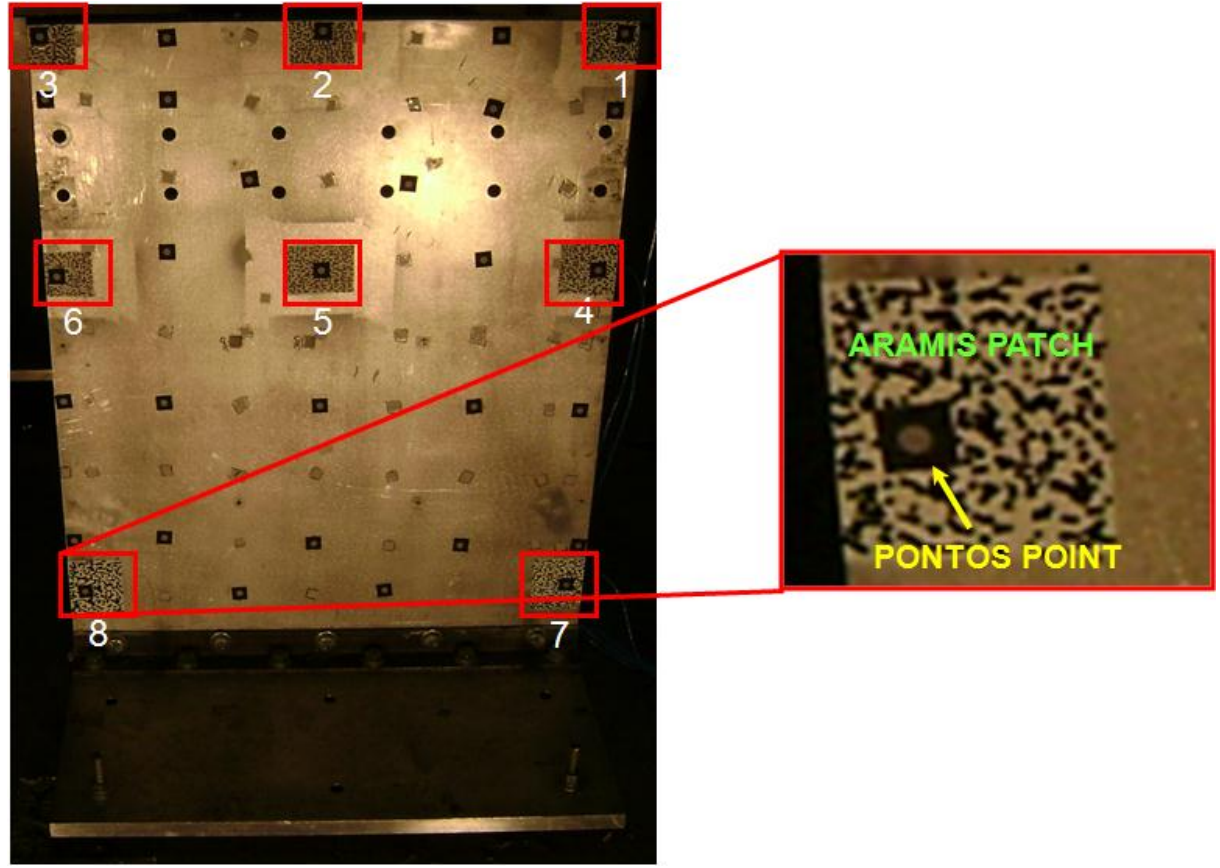

Fig. 10. PONTOS target locations and ARAMIS patches.

a rate of 200 frames per second or $200 \mathrm{~Hz}$. Details of imaging data is not contained herein. Specific processing of this data is contained in [10]; general DIC is presented in [17-19].

\section{Displacement response}

There are three different sensor location configurations studied which compare displacements responses at three different locations in the Upright structure; this is done to show the robustness of the expansion process in the event of a failed sensor. All the sensor configurations use 7 out of the 8 locations (Fig. 10).

1. Sensor set-up 1 - uses locations $2,3,4,5,6,7$ and 8 for expansion.

2. Sensor set-up 2 - uses locations 1, 2, 3, 5, 6, 7 and 8 for expansion.

3. Sensor set-up 3 - uses locations 1, 2, 3, 4, 5, 6 and 8 for expansion.

For the first sensor configuration (Sensor set-up 1), 2, 3, 4, 5, 6, 7 and 8 sensor displacement responses were used in expansion process to expand to 3112 locations of the nodes in the finite element model; note that these 3112 locations are on the upright portion of the base-upright structure. The sensor 1 displacement response recorded experimentally was used for comparison with expanded solution. Note that the impact was made at the location 1 on the Upright structure, so location 1 will see the highest amplitude of response. The comparison of the expanded and the experimentally obtained response is shown in Fig. 11a. The response was recorded for 4 seconds as shown. The responses predicted by the proposed technique compares well with the experimentally recorded response at the top-right location. For comparing the time responses, a TRAC was performed between the expanded solution and experimentally recorded response. A TRAC value of $98 \%$ was observed which again showed a very good agreement between the two solutions. 

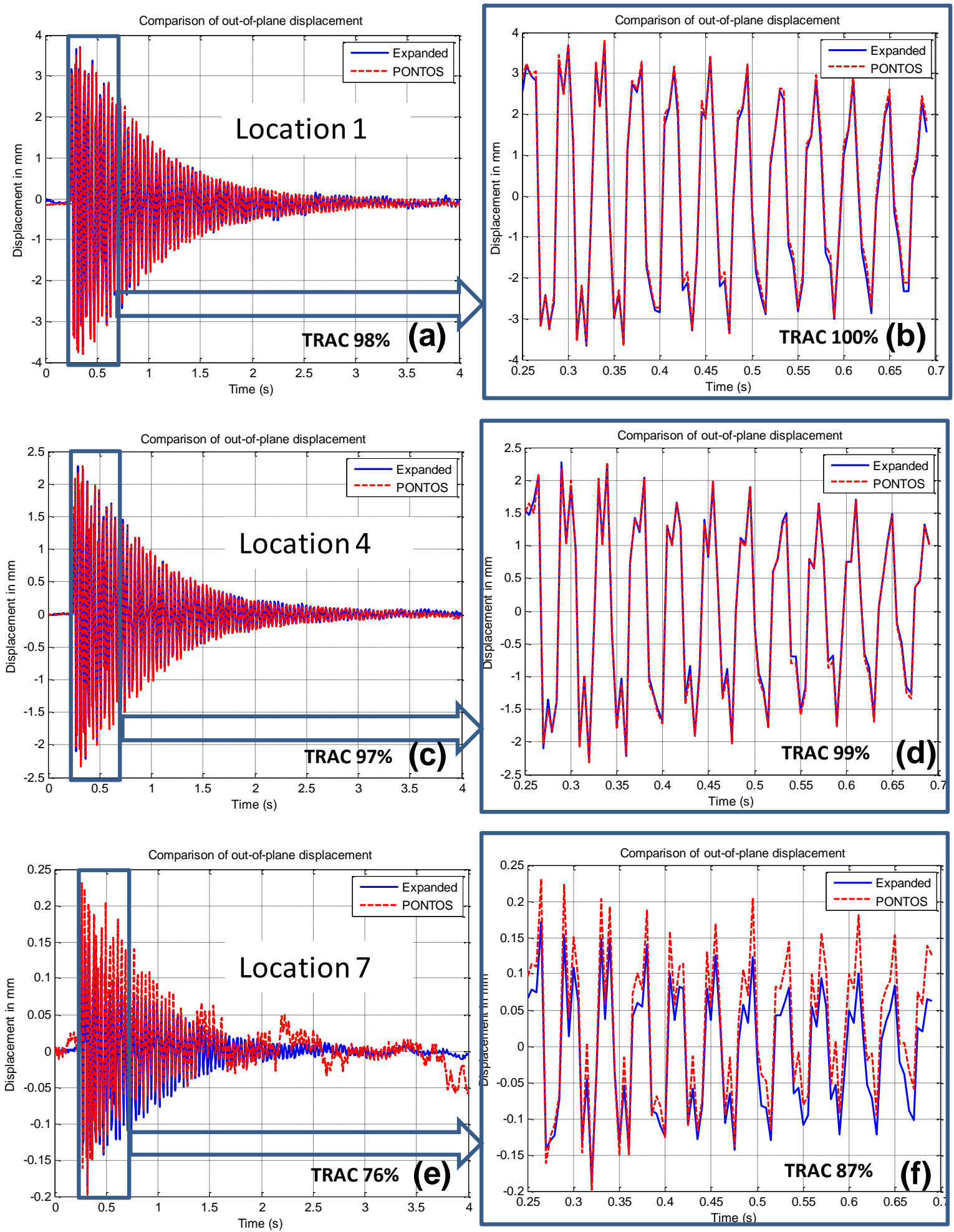

Fig. 11. Overlay of the displacement responses (shown for complete and initial transient period) from predicted solution using first five modes for expansion and PONTOS at three different locations of the BU. 
The transient response comparison for initial time steps (where highest amplitude of response is observed) is sho wn in Fig. $11 \mathrm{~b}$ which is the first $0.5 \mathrm{sec}$ of the response. As seen, the solution predicted by the proposed technique compares well with the experimentally recorded response. Note that the TRAC value increases from $98 \%$ to $100 \%$ because good correlation is observed for high Signal-to-Noise-Ratio (SNR) time period.

The second sensor configuration (Sensor set-up 2) studied was using 1, 2, 3, 5, 6, 7 and 8 sensors excluding the 4th location. The comparison between expanded displacement response (which used the 7 sensors to expand to up to 3112 points in the Upright) and experimentally recorded displacement response at location 4 is made and shown in Fig. 11c. The mode configuration used previously in the expansion process is again used in this case. A good correlation between the expanded and experimentally recorded displacement data set is observed. TRAC of up to 97\% is seen which means that the predicted solution is very similar to the experimentally recorded data set even though the comparison location at Location 4 was half-way to the fixed edge of the structure (where more noise in experimental data set is expected).

Comparison for initial time period as seen in Fig. 11d shows that even for mid-range SNR, the proposed technique does a good job of predicting accurate response. A TRAC of $99 \%$ further substantiates the argument of accurate response prediction.

A third sensor configuration (Sensor set-up 3) was studied wherein sensors 1, 2, 3, 4, 5, 6, and 8 were used excluding the sensor 7 which is very close to the fixed edge of the structure. Higher noise and low SNR is expected. Figure 11e shows comparison between the predicted solution and experimentally recorded displacement response at location 7. As expected the experimental response is very noisy, especially for time period 2-4 seconds where the response dies out. A TRAC of $76 \%$ is observed for the time response comparison between the predicted and the experimental responses over the full range of 4 seconds.

A TRAC value of $87 \%$ was obtained for the time responses at initial time steps where higher response was expected. A fairly good correlation is observed in Fig. 11f between the experimental and the predicted responses.

For sensor configuration 1, the output response at locations other than top right corner were also collected and compared with expanded results. Note however that the measurements used for comparison purposes were not included in expansion process. The output response comparison plots are shown in Fig. 12 at five such locations.

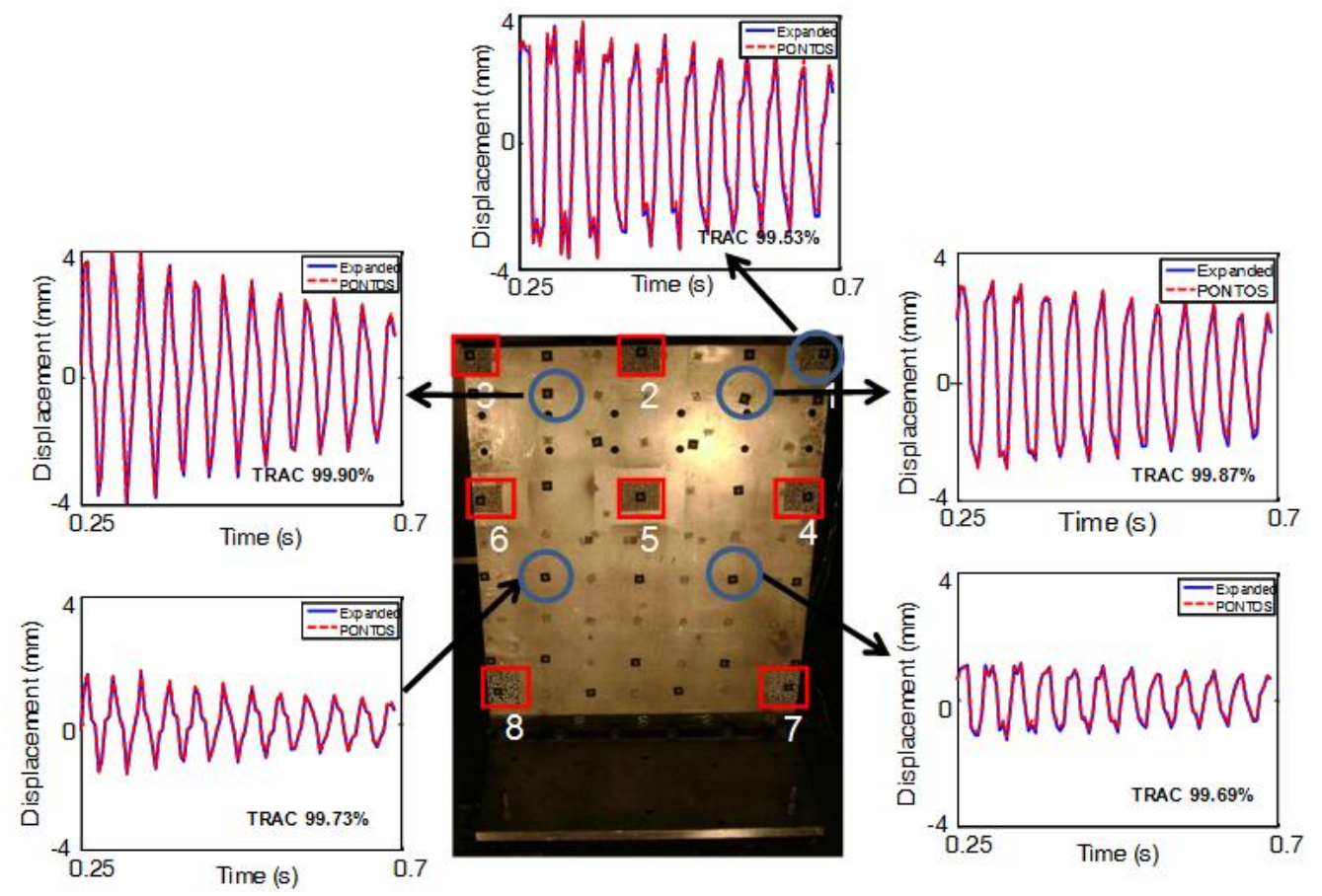

Fig. 12. Overlay of the displacement responses from predicted solution using first five modes for expansion and PONTOS at five different locations; expansion performed using sensor configuration 1 . 


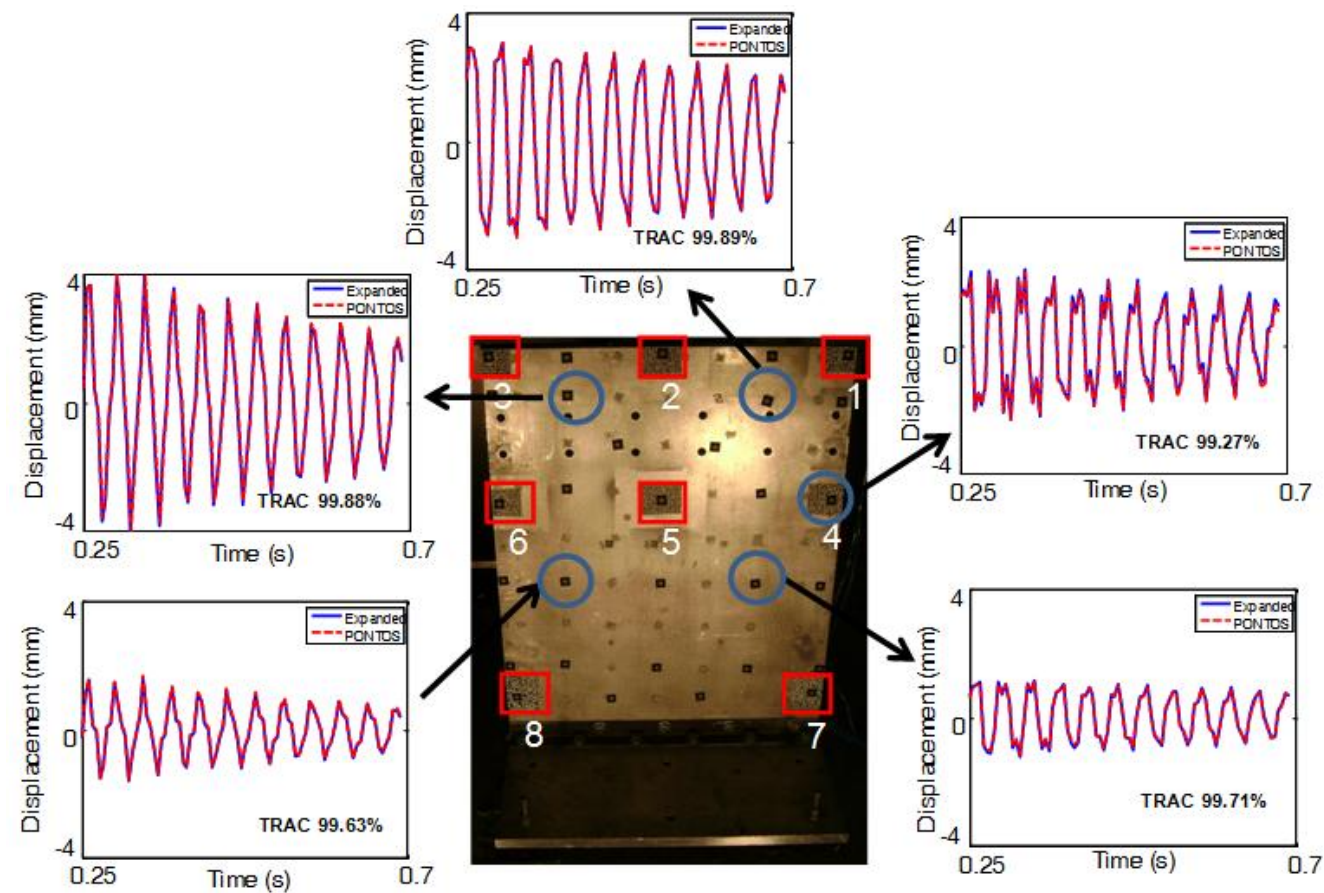

Fig. 13. Overlay of the displacement responses from predicted solution using first five modes for expansion and PONTOS at five different locations; expansion performed using sensor configuration 2.

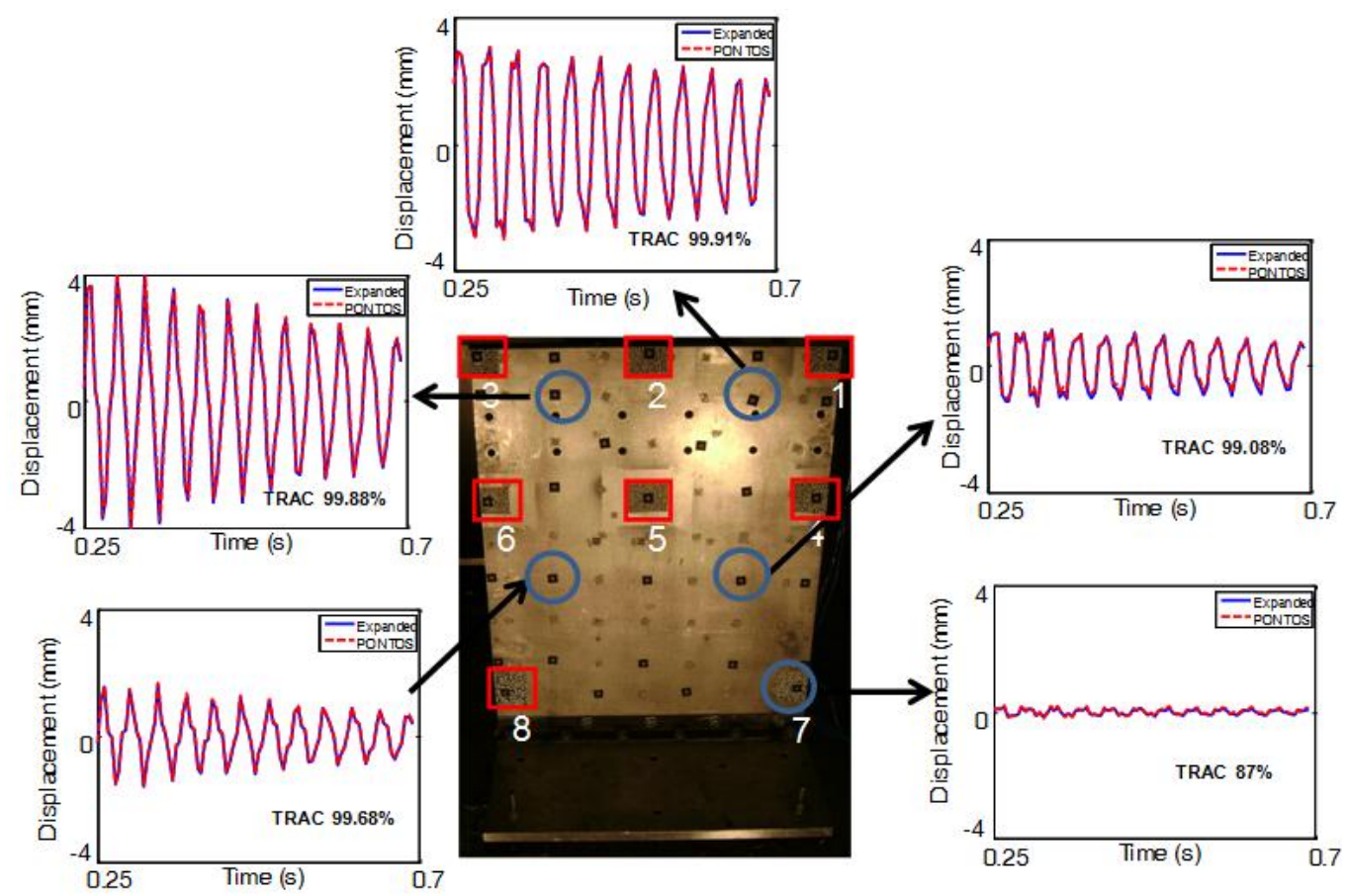

Fig. 14. Overlay of the displacement responses from predicted solution using first five modes for expansion and PONTOS at five different locations; expansion performed using sensor configuration 3. 
Notice that at all locations the TRAC correlation values are always higher than 99\% which indicates an excellent correlation of expanded data with measurements for the time period shown.

With the second sensor configuration, output response at locations other than location 4 were also collected and compared with the expanded results. Again the measurements used for comparison purposes were not included in expansion process. The output response comparison plots are shown in Fig. 13 at five such locations. Notice that at all but one location the TRAC correlation values are always higher than $99 \%$ which indicates a very good correlation of expanded data with measurements for the time period shown. This confirms that even with 7 sensors, accurate prediction of response is possible using proposed methodology for up to more than 3000 locations.

Figure 14 shows the expanded output responses at five different locations in comparison with the reference experimental measurements for third sensor configuration. The measurements used for comparison purposes were not included in expansion process. Notice that at all locations except the location 7, the TRAC correlation values are always higher than $99 \%$ which indicates a very good correlation of expanded data with measurements for the time period shown. For the 7th location, which is near to the fixed base of the structure, the displacement response is very small as compared to other locations as seen in Fig. 14. The Signal-to-Noise-Ratio (SNR) is much smaller and hence the TRAC correlation drops to $87 \%$.

Overall, each of the different configurations studied provided very good expanded data.

\section{Strain response}

The strain response is obtained from full-field expanded displacements for all the three sensor configurations discussed in Case 1 using strain recovery process outlined in [10,21,22]. The strain response has been calibrated for static load and discrete sine test.

Ideally for comparing the strain response obtained using the proposed methodology, the reference solution should be obtained experimentally. However, the minimum strain levels measured by using the DIC technique is 50 micro-strain. The maximum strain levels obtained in BU were well below the minimum strain levels that the DIC can measure accurately, even in regions where the highest strain resulted. So for comparison purposes, a FEM of the BU was used. A force pulse was applied to the FEM of the BU at the location where the actual impact was made during the experimental testing. The BU FEM has shown good correlation for previous studies performed for the purpose of extracting mode shape information. Also the forcing pulse used for exciting the BU FEM was one of the impact force measurements made during the impact tests. The forcing pulse as shown in Fig. 15 was discretized at every 0.0001 seconds. As seen in Fig. 15, the impact on the BU was made at 0.25 seconds.

\section{DIGITIZED IMPULSIVE EXCITATION}

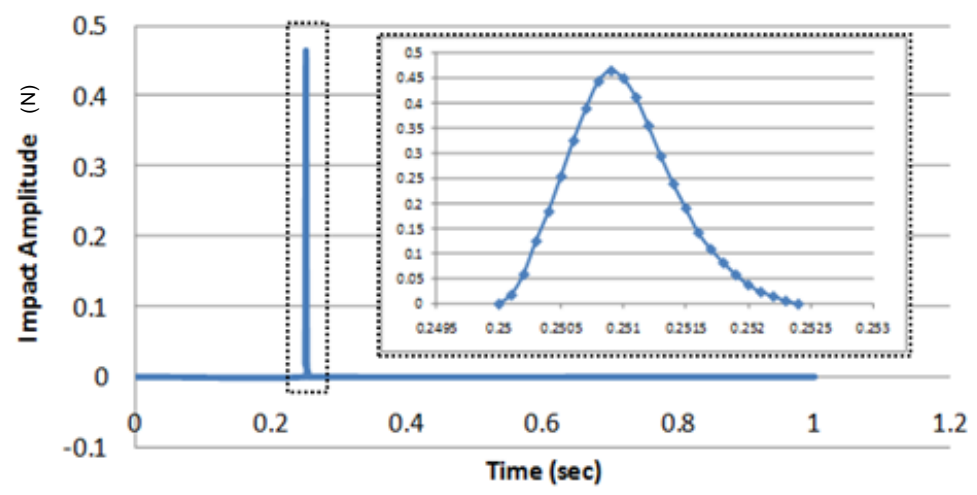

Fig. 15. Forcing pulse used for BU impact.

Using Abaqus, a direct integration approach was used for obtaining the strain solutions for the FEM. The comparison between the FEA strain solution and the strain solution obtained using the proposed technique is made in 

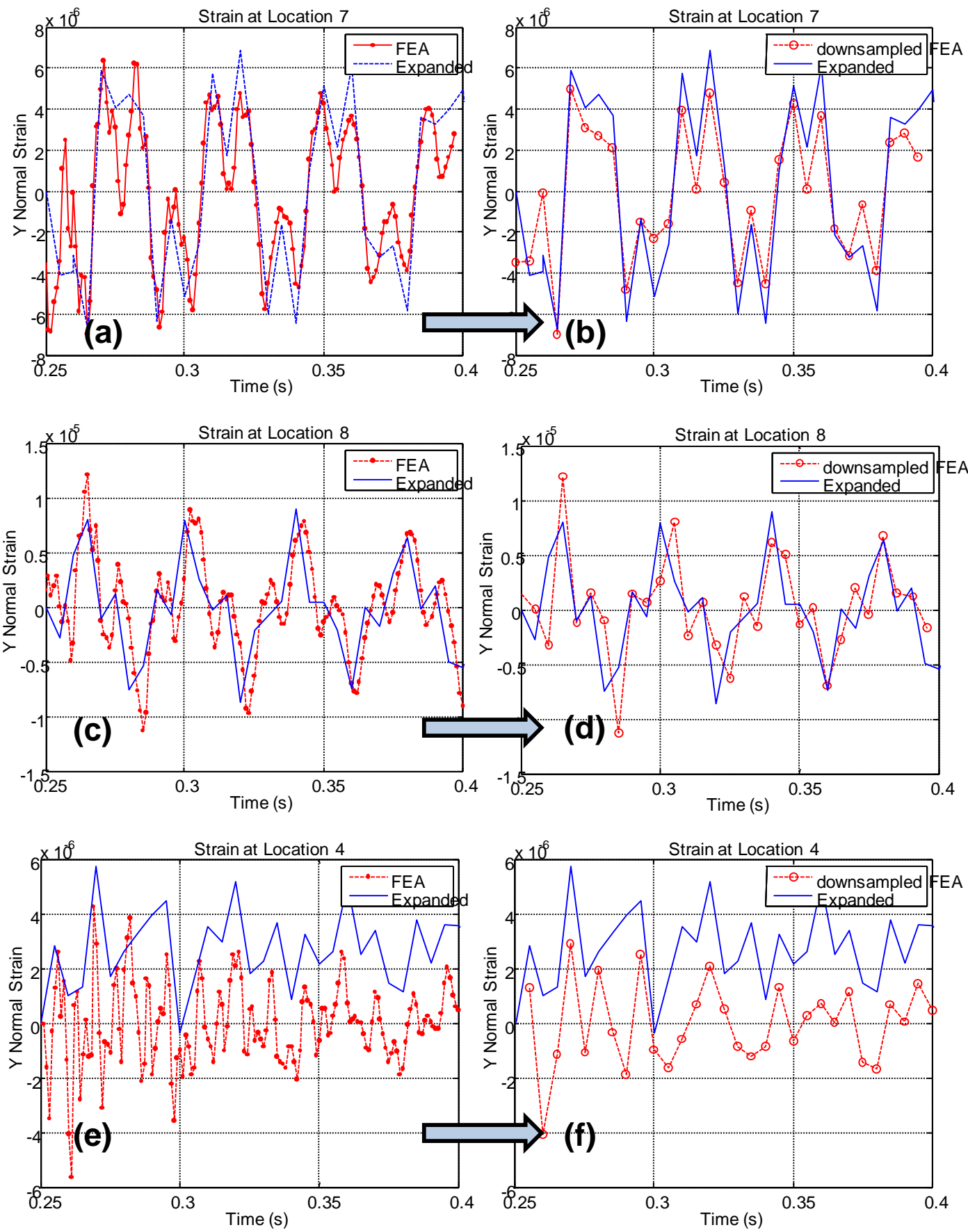

Fig. 16. Comparison of strain response from FEA solution and predicted solution using direct integration of the equations of motion with (a,c,e) contain finer step and $(b, d, f)$ contain identical time step.

Figs 16a, c and e at locations 7, 8 and 4 respectively in the BU. The response shown is after the impact was made at 0.25 seconds for a sample time period of 0.15 seconds. The FEA transient solution was obtained for 400 steps with 0.001 seconds per step. The strain solution obtained from the proposed technique uses the full-field displacement solution. So the displacement sampling rate of $0.005 \mathrm{sec}$ per sample also applies for the strain obtained from 

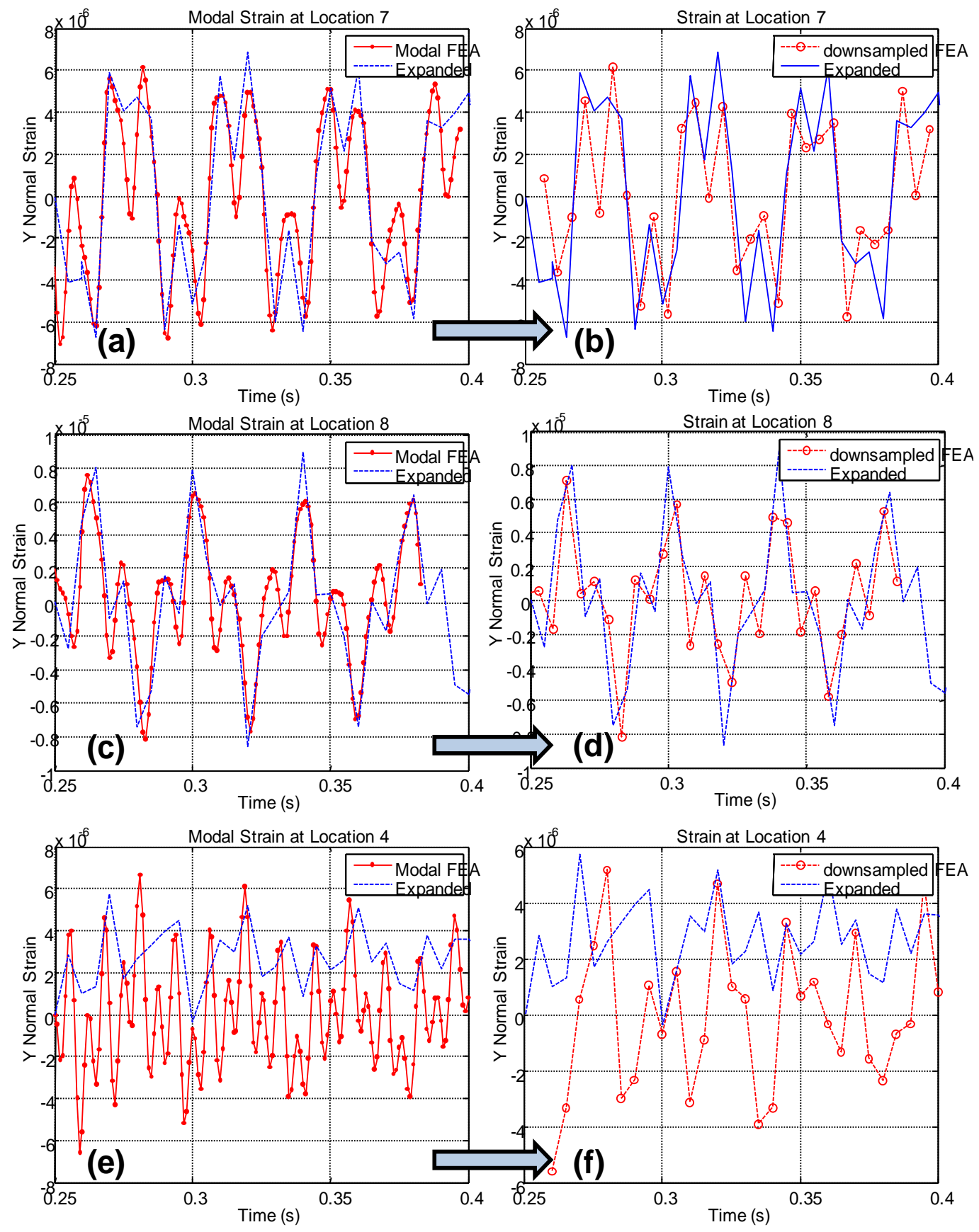

Fig. 17. Comparison of strain response from FEA solution and predicted solution using a mode superposition approach with $(a, c, e)$ contain finer step and $(b, d, f)$ contain identical time step.

proposed technique. The FEA solution was performed at a finer time step to properly obtain an accurate numerical solution. This implies that the FEA and test data have different time increments. The FEA strain response had to be decimated (or down-sampled) to the response data points recorded during experimentation. The comparison of this decimated FEA strain solution with the predicted strain response using the expansion technique is shown in Figs 16b, $\mathrm{d}$ and $\mathrm{f}$ for locations 7,8 and 4 respectively. 
The FEA solution shows contribution of higher order modes because the direct integration scheme was used to obtain the solution. However, a good overall agreement is observed between the predicted and FEA strain solutions for locations 7 and 8 which are higher strain regions in the structure as they are closer to the fixed base. Location 4, which is at the mid-level of the BU does not see as much strain in comparison to locations 7 and 8 . There is a slight DC bias that appears on the data but the general trend is reasonable considering the lower level of strain observed at this location.

Clearly, there is some small contribution of the higher order modes at the very start of the transient. In order to understand the effect of using less modes for the response, a mode superposition solution was performed using only the first five modes used in the expansion process; this essentially truncates the effects of the higher order modes by using the modal superposition solution scheme. The results after using modal superposition scheme instead of the direct integration approach are shown in Fig. 17. Again, the down-sampled results have also been shown at the three comparison locations (Location 7, 8 and 4) in Fig. 17. Use of modal superposition solution scheme for obtaining FEA strains, does not affect the differences between the strain responses at location 4 . However, at locations 7 and 8 , as seen from Figs $17 \mathrm{~b}$ and d, there is definite improvement observed in the correlation between the FEA strains and the strains predicted through the proposed approach because the effect of higher order modes is not observed in the FEA solution as only the first five modes were used in the mode superposition solution scheme.

Location 4 was shown to provide excellent displacement response results as compared to the location 7 . Note that the location 4 is nearer to the free edge of the BU structure, while the locations 7 and 8 are very near to the fixed edge of the BU structure. Invariably, higher displacement response and lower strain response will be observed at location 4 as compared to locations 7 and 8 . The displacement response at location 7 was contaminated by experimental noise as observed in displacement case. Similarly, there is possibility of the strain response at location 4 to be contaminated by noise hence producing the discrepancies observed. However the locations 7 and 8 provide excellent strain response comparison, which validates the proposed methodology of estimating dynamic -strain experimentally.

\section{Concluding remarks}

The prediction of full field dynamic response along with full field dynamic stress-strain is an important item in considering the structural health of a structure and the cumulative overall fatigue life of a structure. Conventional modeling approaches can fall short in the identification of the actual loads and actual boundary conditions for many situations.

A new approach that utilizes the response at limited sets of measurement locations in conjunction with orthogonal expansion functions to identify the full field displacement are presented. This full field displacement is then used in conjunction with the finite element back substitution process to obtain full field dynamic strain - all without the specific identification of loads or boundary conditions.

Analytical cases presented in the paper illustrate the technique and identify the need for appropriate number of modes to identify the displacement response; the number of modes necessary is dependent on the actual number of modes that are excited by the forces on the system as is the case in any dynamic response application. The studies also showed that, in general, more modes were needed to converge to an accurate strain result but this was not a limitation in the process. Further study is needed to better understand the need for additional modes.

Experimental data was also used with the proposed methodology and the results followed the findings of the analytical studies performed. A finite element model was used with the measured experimental data to verify and validate the displacement and strain results obtained. Overall very good correlation resulted. The robustness of the technique was demonstrated with subsets of measured data to show that the process could predict accurate results.

\section{Acknowledgments}

This work was supported by the U.S. Army Research Office Nanomanufacturing of Multifunctional Sensors Ref. Award Number: W911NF-07-2-0081 and the National Science Foundation under Grant No. 0900534, entitled "Dynamic Stress-Strain Prediction of Vibrating Structures in Operation". Any opinions, findings, and conclusions or recommendations expressed in this material are those of the authors and do not necessarily reflect the views of the National Science Foundation or Army Research Office. 


\section{Appendix A. Model reduction and expansion procedure}

In order to develop the SEREP (System Equivalent Reduction-Expansion Process) process used here, model reduction needs to be formulated first, as this is the basis of the expansion process. Essentially, the relationship between the full space finite element model and the very limited set of measured degrees of freedom (DOF) needs to be formulated. This transformation matrix is then the necessary piece to relate the test measured points to the finite element nodes.

To reduce a full set of ' $n$ ' finite element DOF to a set of ' $a$ ' master or test DOF, a transformation relationship must be determined. This transformation can be written in general terms as

$$
\left\{x_{n}\right\}=\left\{\begin{array}{c}
x_{a} \\
x_{d}
\end{array}\right\}=[T]\left\{x_{a}\right\}
$$

The subscript ' $d$ ' refers to the deleted DOF and $[T]$ is the transformation matrix. Note that the master set of ' $a$ ' DOF will typically refer to the set of test DOF. Due to energy conservation, the mass matrix $[M]$ and stiffness matrix $[K]$ can be reduced according to

$$
\begin{aligned}
& {\left[M_{a}\right]=[T]^{T}\left[M_{n}\right][T]} \\
& {\left[K_{a}\right]=[T]^{T}\left[K_{n}\right][T]}
\end{aligned}
$$

The $[T]$ matrix is developed for the SEREP process in such a way that an eigensolution of the resultant reduced mass and stiffness matrices produces the exact frequencies and mode shapes when compared to that of the full space model. To develop the SEREP [T] matrix, the modal transformation matrix must be partitioned into the set of master 'a' DOF and the ' $n$ ' full space DOF according to

$$
\left\{x_{n}\right\}=\left\{\begin{array}{c}
x_{a} \\
x_{d}
\end{array}\right\}=\left[\begin{array}{c}
U_{a} \\
U_{d}
\end{array}\right]\{p\}
$$

The relationship for only the 'a' DOF is then written as

$$
\left\{x_{a}\right\}=\left[U_{a}\right]\{p\}
$$

Assuming that the number of modes is less than that of the number of ' $a$ ' DOF, (A-5) can be shown to be manipulated while using the generalized inverse to obtain

$$
\{p\}=\left(\left[U_{a}\right]^{T}\left[U_{a}\right]\right)^{-1}\left[U_{a}\right]^{T}\left\{x_{a}\right\}=\left[U_{a}\right]^{g}\left\{x_{a}\right\}
$$

This result can be substituted back into the normal modal transformation to obtain

$$
\left\{x_{n}\right\}=\left[U_{n}\right]\left[U_{a}\right]^{g}\left\{x_{a}\right\}=\left[T_{u}\right]\left\{x_{a}\right\}
$$

The SEREP transformation matrix is therefore defined as

$$
\left[T_{U}\right]=\left[U_{n}\right]\left[U_{a}\right]^{g}
$$

\section{Appendix B. Correlation tools}

The correlation tools utilized to compare the results of the expanded RTO (Real Time Operating) [ERTOn] and "reference time solution" [REFn] are briefly discussed here.

The MAC (Modal Assurance Criteria) [9] as used for this work will identify the correlation of the expanded real time operating displacement solution obtained with the reference solution. The MAC can be computed at each time 
step $\mathrm{t}$ to compare the transient displacement solution with time. The MAC is written as

$$
M A C_{-} R T O=\frac{\left[\{R E F n\}^{T}\{\text { ERTOn }\}\right]^{2}}{\left[\{R E F n\}^{T}\{R E F n\}\right]\left[\{\text { ERTOn }\}^{T}\{\text { ERTOn }\}\right]}
$$

Similar to the MAC, the TRAC (Time Response Assurance Criterion) is a tool used to determine the degree of correlation between two time traces. For the cases presented here, the TRAC is the correlation for one DOF over all time for the expanded time data [ERTOn] compared to the actual measured data [REFn]. The TRAC is written as

$$
\text { TRAC_RTO }=\frac{\left[\{\operatorname{REFn}(t)\}^{T}\{\operatorname{ERTOn}(t)\}\right]^{2}}{\left[\{\operatorname{REFn}(t)\}^{T}\{\operatorname{REFn}(t)\}\right]\left[\{\operatorname{ERTOn}(t)\}^{T}\{\operatorname{ERTOn}(t)\}\right]}
$$

The values produced by both the MAC and TRAC will range from 0 to 1 ; values approaching 1 indicate good correlation.

Also used for comparing the [ERTOn] with reference time solution is an absolute different between the two solutions at each time step. The magnitude of the difference is a measure of how much the [ERTOn] deviates from the reference time solution.

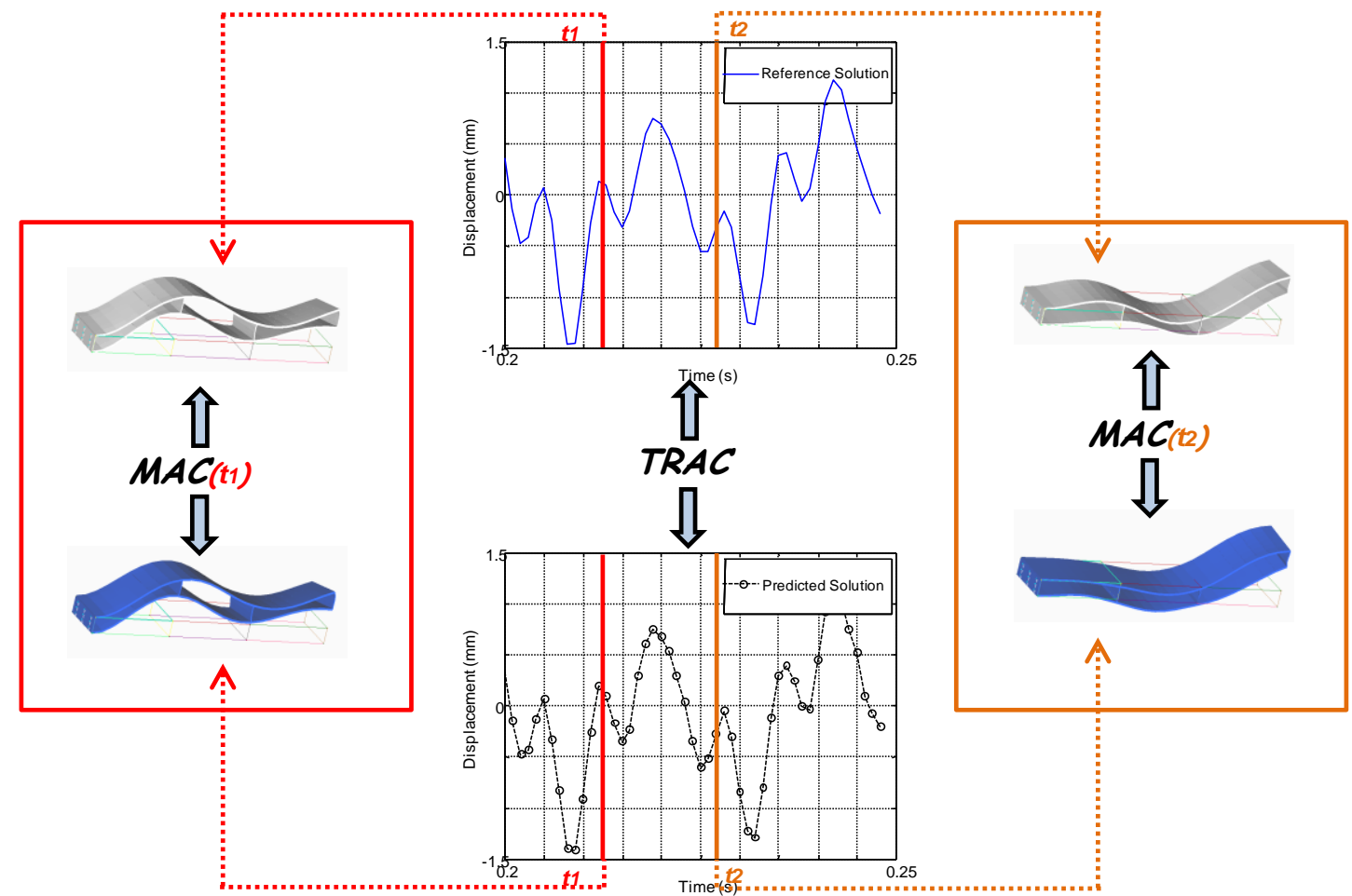

Fig. B-1. Schematic showing MAC and TRAC.

\section{Appendix C. Correlation results for upright structure}

The upright structure used for the experimental studies presented herein has been subjected to many different structural dynamic tests and correlated to the finite element model used in this study. The actual structure with 
measurement locations identified is shown along with a typical drive point FRF in Fig. C-1. The finite element model with the first 20 mode shapes are shown in Fig. C-2; the model was bolted to the floor and constrained at those locations.
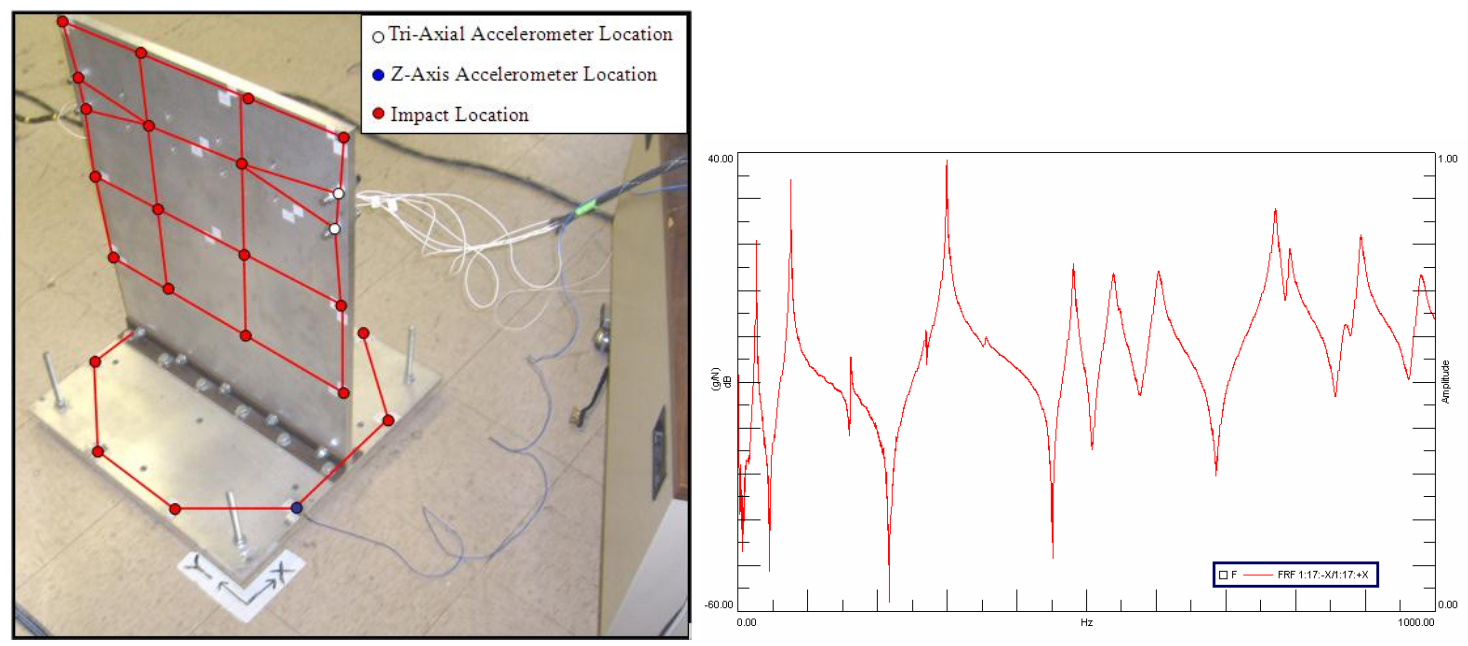

Fig. C-1. Upright test structure with measurement locations along with a typical drive point FRF.
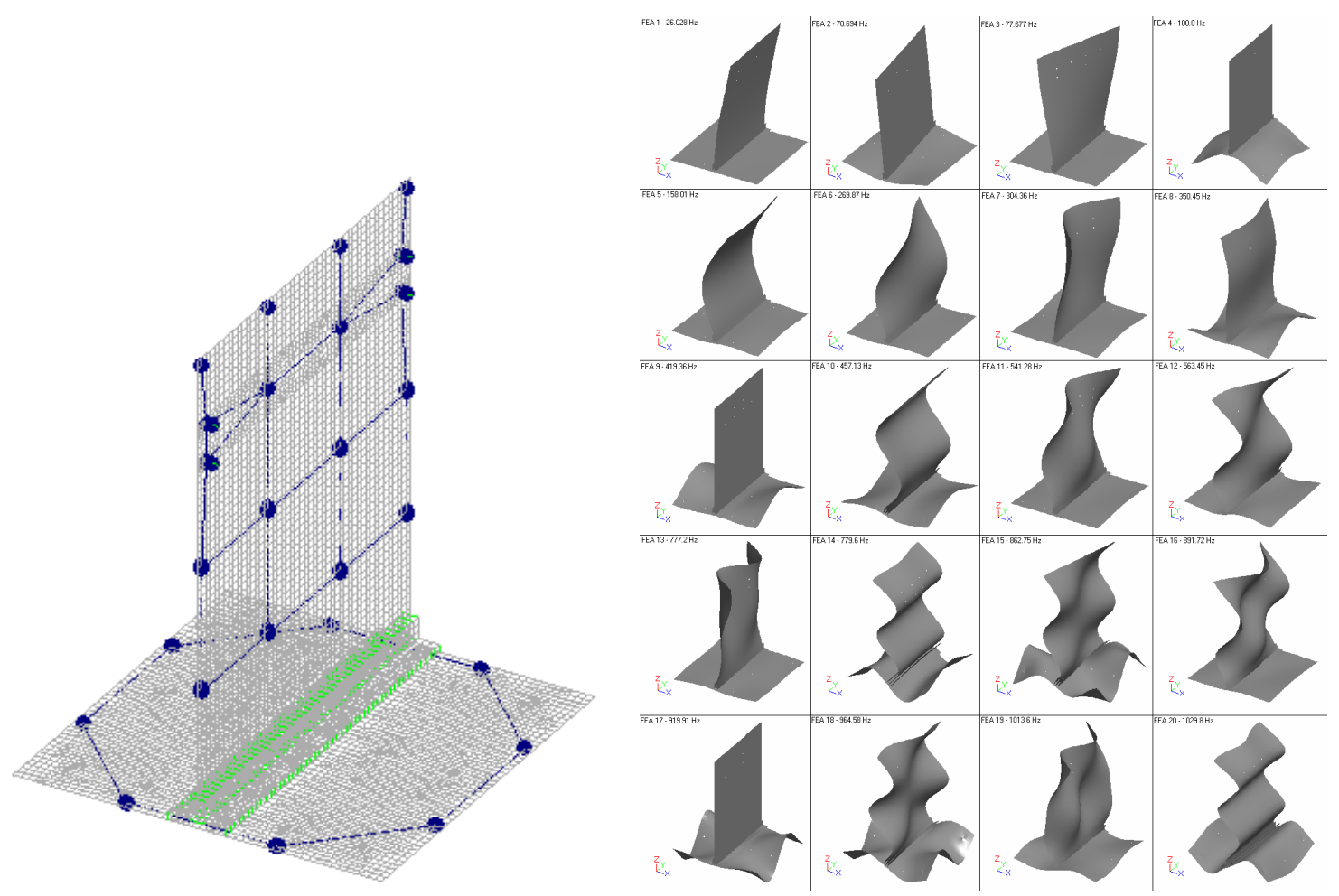

Fig. C-2. Upright finite element model with first 20 mode shapes.

An experimental modal test was performed and frequencies and mode shapes were extracted using multiple reference modal parameter estimation strategies. The resulting experimental data was correlated to the finite ele- 
ment model and the results of the correlation are shown in Table C-1. Note that the lower order modes that are used in the studies in this work correlate very well with very high MAC values as well as very high POC (pseudo-orthogonality check) values.

Table C-1

Correlation of upright test data to FEA model

\begin{tabular}{ccccccc}
\hline $\begin{array}{c}\text { FEA mode } \\
\text { number }\end{array}$ & $\begin{array}{c}\text { Experimental } \\
\text { mode number }\end{array}$ & $\begin{array}{c}\text { FEA fre- } \\
\text { quencies (Hz) }\end{array}$ & $\begin{array}{c}\text { Experimental } \\
\text { frequencies (Hz) }\end{array}$ & $\begin{array}{c}\text { Frequency } \% \\
\text { difference }\end{array}$ & $\begin{array}{c}\text { MAC } \\
\text { value } \\
(\%)\end{array}$ & $\begin{array}{c}20 \text { mode } \\
\text { POC } \\
\text { value }\end{array}$ \\
\hline 1 & 1 & 26.03 & 26.17 & -0.54 & 99.8 & 1.071 \\
2 & 2 & 70.70 & 67.34 & 4.75 & 98.5 & 0.973 \\
3 & 3 & 77.69 & 75.89 & 2.32 & 99.3 & 1.033 \\
4 & 4 & 108.80 & 104.48 & 3.97 & 97.1 & 1.073 \\
5 & 5 & 158.01 & 161.55 & -2.24 & 98.9 & 1.048 \\
6 & 6 & 269.88 & 270.00 & -0.04 & 98.9 & 1.019 \\
7 & 7 & 304.40 & 299.93 & 1.47 & 96.5 & 1.024 \\
8 & 8 & 350.45 & 354.60 & -1.18 & 98.5 & 0.979 \\
9 & 9 & 419.36 & 419.22 & 0.03 & 94.3 & 0.483 \\
10 & 10 & 457.13 & 482.15 & -5.47 & 92.7 & 0.836 \\
11 & 12 & 541.29 & 547.84 & -1.21 & 96.6 & 0.995 \\
12 & 11 & 563.47 & 538.20 & 4.48 & 86.3 & 0.785 \\
13 & 13 & 777.29 & 771.76 & 0.71 & 90.3 & 1.002 \\
14 & 14 & 779.61 & 791.18 & -1.48 & 97.7 & 0.944 \\
15 & 15 & 862.76 & 871.90 & -1.06 & 84.3 & 0.795 \\
16 & 16 & 891.73 & 893.97 & -0.25 & 96.2 & 1.041 \\
17 & 17 & 919.91 & 953.24 & -3.62 & 64.2 & 0.506 \\
18 & 18 & 964.61 & 979.99 & -1.59 & 95.7 & 0.960 \\
19 & 19 & 1013.58 & 1027.71 & -1.39 & 96.5 & 0.912 \\
20 & 20 & 1029.81 & 1075.56 & -4.44 & 95.4 & 1.021 \\
\hline
\end{tabular}

Additional correlation studies and additional information on the structure can be found in [23,24].

\section{References}

[1] P. Avitabile, F. Piergentili and K. Lown, Identifying Dynamic Loadings from Measured Response, Sound and Vibration, August 1999.

[2] C. Chipman, Expansion of Real Time Operating Data, Master's Thesis, University of Massachusetts Lowell, May 2009.

[3] C. Chipman and P. Avitabile, Expansion of Real Time Operating Data for Improved Visualization, Proceedings of the Twenty-Sixth International Modal Analysis Conference, Orlando, FL, Feb 2008

[4] C. Chipman and P. Avitabile, Expansion of Transient Operating Data, Proceedings of the Twenty-Seventh International Modal Analysis Conference, Orlando, FL, Feb 2009.

[5] R.J. Guyan, Reduction of Stiffness and Mass Matrices, AIAA Journal, Vol. 3, No 2, 1965.

[6] M. Paz, Dynamic condensation, AIAA Journal 22(5) (May 1984).

[7] J.C. O'Callahan, P. Avitabile and R. Riemer, System Equivalent Reduction Expansion Process, Seventh International Modal Analysis Conference, Las Vegas, Nevada, February 1989.

[8] D.C. Kammer, A hybrid approach to test analysis model development for large space structures, Journal of Vibration and Acoustics 113 (July 1991)

[9] R.J. Allemang and D.L. Brown, A Correlation Coefficient for Modal Vector Analysis, First International Modal Analysis Conference, Orlando, Florida, November 1982, pp. 110-116.

[10] P. Pingle, Prediction of Full-Field Dynamic Stress-Strain from Limited Sets of Measured Displacement Data, Doctoral Thesis, University of Massachusetts Lowell, August 2010.

[11] MATLAB R2008b - The MathWorks, Natick, Massachusetts.

[12] FEMAP - Finite Element Modeling And Postprocessing, Version 9.3.1, Copyright @ 2007 UGS Corp.

[13] ARAMIS, v. 6.0 User's Manual, GOM mbH, Braunschweig, Germany, 2007.

[14] PONTOS, User's Manual, Revision A, GOM mbH, Braunschweig, Germany, 2004.

[15] FEMtools 3.0 - Dynamic Design Solutions, Leuven, Belgium.

[16] ABAQUS 6.5.1 @ Dassault Systèmes, 2004. 
[17] E. Mikhail, J. Bethel and J. McGlone, Introduction to Modern Photogrammetry, John Wiley and Sons, 2001.

[18] B. Triggs, A. Zisserman and R. Szeliski, in: Bundle Adjustment - A Modern Synthesis, Triggs, Bill, Philip McLauchlan, Richard Hartley, and Andrew Fitzgibbon, eds, Vision Algorithms'99, LNCS 1883, 2000, pp. 298-372.

[19] M. Helfrick, An Investigation of 3D Digital Image Correlation for Structural Health Monitoring and Vibration Measurement, Master's Thesis, University of Massachusetts Lowell, 2008.

[20] P. Pingle and P. Avitabile, Prediction of Full Field Dynamic Stress/Strain from Limited Sets of Measured Data, Proceedings of the Twenty-Eighth International Modal Analysis Conference, Jacksonville, FL, Feb 2010.

[21] P. Pingle and P. Avitabile, Full Field Dynamic Stress/Strain Estimation From Limited Sets of Measured Data, Proceedings of the Twenty-Ninth International Modal Analysis Conference, Jacksonville, FL, Feb 2011.

[22] P. Pingle and P. Avitabile, Limited Experimental Displacement Data Used for Obtaining Full-field Dynamic Stress/Strain, Proceedings of the Twenty-Ninth International Modal Analysis Conference, Jacksonville, FL, 2011.

[23] P. Pingle, J. Sailhamer and P. Avitabile, Comparison of 3D Laser Vibrometer and Accelerometer Frequency Measurements, Proceedings of the Twenty- Seventh International Modal Analysis Conference, Orlando, FL, 2009.

[24] C. Warren, P. Pingle, C. Niezrecki and P. Avitabile, Comparison of Image Based, Laser, and Accelerometer Measurements, Proceedings of the Twenty-Eighth International Modal Analysis Conference, Jacksonville, FL, 2010. 

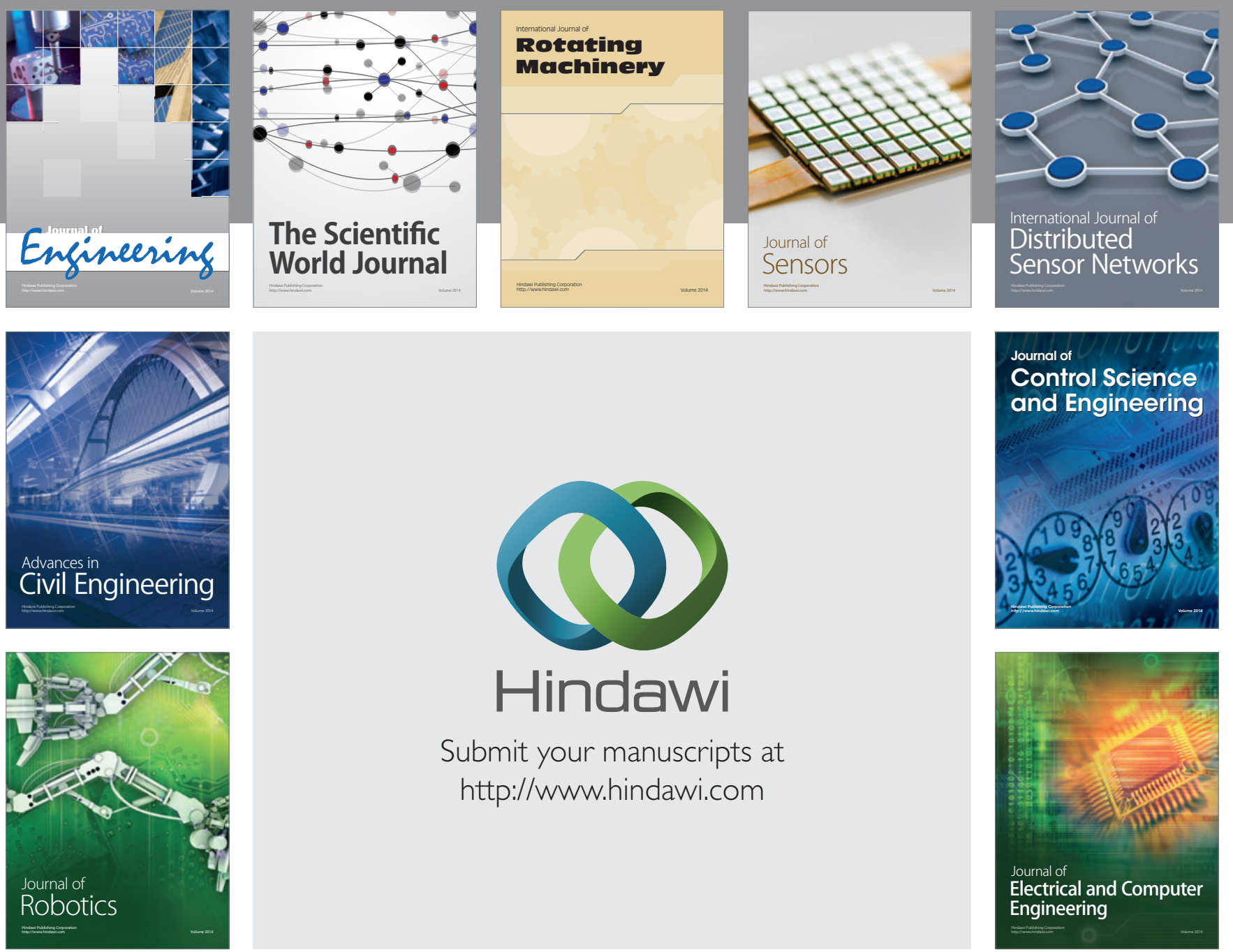

Submit your manuscripts at

http://www.hindawi.com
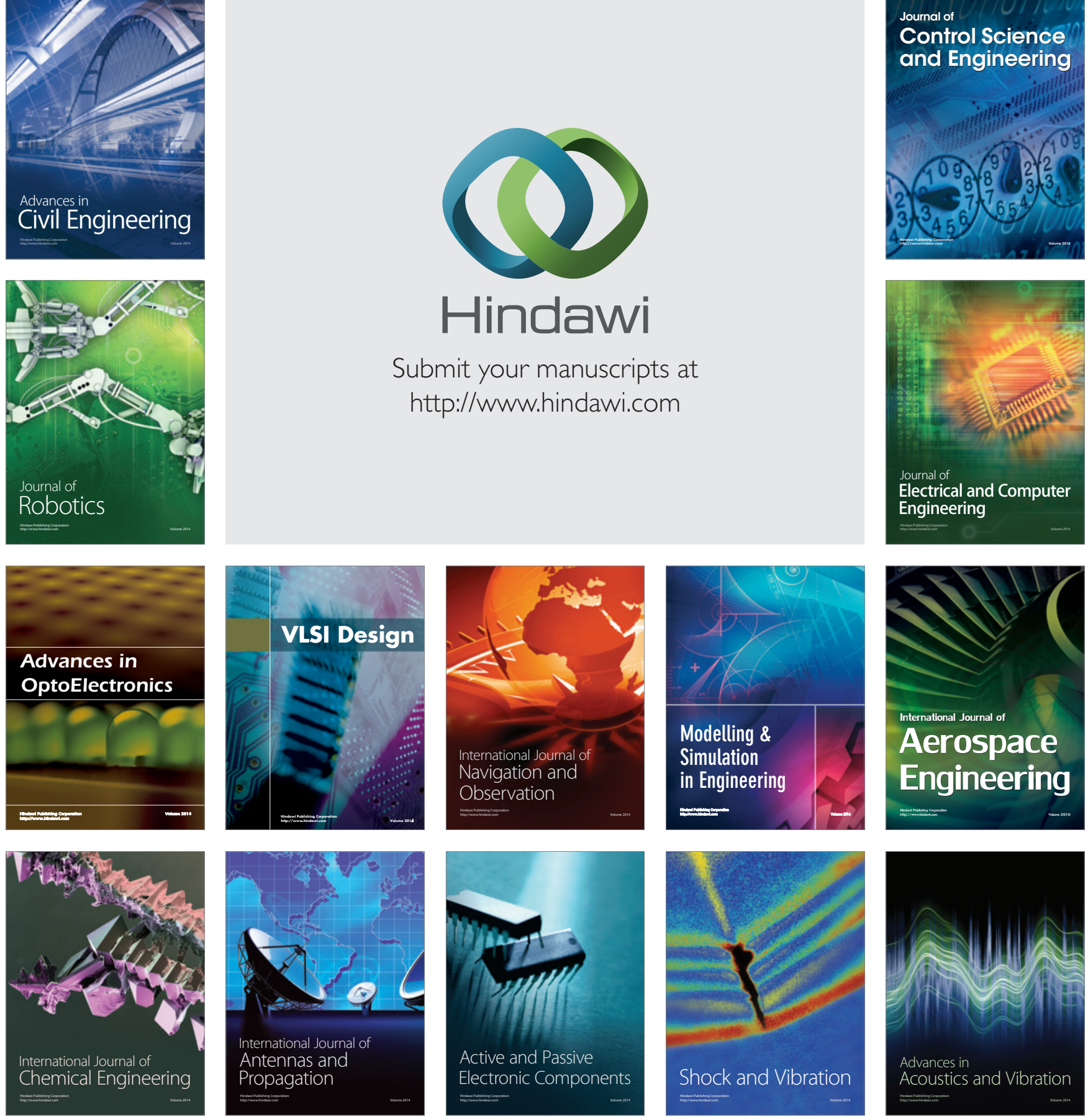